\title{
Development of titanium dioxide nanotube-based arrays for the electrocatalytic degradation and electrochemical detection of emerging pharmaceuticals in water
}

\author{
K. Carlson ${ }^{1}$, J. Tamllos ${ }^{2}$, A. Timmerman ${ }^{1}$, M. Misra ${ }^{1,2}$ \\ \& S. Mohanty ${ }^{1,2}$ \\ ${ }^{1}$ Department of Metallurgical Engineering, University of Utah, USA \\ ${ }^{2}$ Department of Chemical Engineering, University of Utah, USA
}

\begin{abstract}
The electrocatalytic degradation and electrochemical detection of ibuprofen (IBU) in water was performed using titanium dioxide nanotube $\left(\mathrm{TiO}_{2}-\mathrm{NT}\right)$ arrays. IBU solutions with starting concentrations of $30 \mathrm{ppm}$ were degraded by $50 \%$ in $15 \mathrm{~min}$ using a $\mathrm{TiO}_{2}-\mathrm{NT}$ array annealed in a reducing atmosphere. Inactivation of E.Coli 25922 was used to determine the radical species generated during degradation in both flow and batch reactors. Semi-quantitative radical concentrations were obtained by using a UV-Vis spectrophotometer to monitor the color change of an oxidation sensitive DPD dye. Electrochemical detection limits of 4 ppb IBU in 50 ppm $\mathrm{NaCl}$ were obtained using a gold coated $\mathrm{TiO}_{2}-\mathrm{NT}$ array annealed in oxygen. These results demonstrated the feasibility of a combined system that could be deployed for inline effluent treatment as these types of systems are robust, chemical free and could be automated for remote control.

Keywords: titanium dioxide nanotubes, emerging contaminants, electrocatalytic degradation.
\end{abstract}

\section{Introduction}

Pharmaceuticals and personal care product (PPCPs) have been detected in wastewater effluents since the late 1960s (Snyder et al. [1]). Wastewater treatment plants (WWTPs) do not currently have requirements for the removal of PPCPs, although a high percentage of many of these chemicals are removed during typical 
treatment processes (Boleda et al. [2]). However, removal is not the equivalent of degradation, as chemicals trapped in the sewage sludge can enter the environment when it is used as crop fertilizer (Clarke and Smith [3]). A compilation of published literature and national studies were found to have detected pharmaceuticals in surface and groundwater at levels less than $100 \mathrm{ng} / \mathrm{L}$, and at levels below $50 \mathrm{ng} / \mathrm{L}$ in drinking water.

One of the most effective methods reported for the degradation of PPCPs was the use of advanced oxidation processes (AOPs), followed by activated carbon or membrane processes (Clarke and Smith [3]). Full-scale industrial processes typically use an ozone-based AOP, which directly attacks double bonds, activated aromatic rings, and hetero-atoms. While effective, its most powerful function is its decomposition into highly reactive and non-selective hydroxyl radicals $(\mathrm{OH} \bullet)$. Some drugs, such as ibuprofen, show little reactivity to ozone treatments; however, significant degradation is achieved when exposed directly to hydroxyl radicals (Boleda et al. [2]). From an ecological standpoint, achieving complete degradation and/or removal is important to prevent the release of compounds or harmful degradation-by-products (DBPs) that exhibit low biodegradability, leading to bioaccumulation and extended environmental exposure.

The use of $\mathrm{TiO}_{2}$ for photocatalytic oxidation of chemical pollutants is well documented (Cesaro et al. [4]). We have recently demonstrated that defect-laden titanium dioxide nanotube $\left(\mathrm{TiO}_{2}-\mathrm{NT}\right)$ arrays are capable of generating hydroxyl radicals under an applied bias in the absence of illumination (Carlson et al. [5]). The following report describes the use of $\mathrm{TiO}_{2}-\mathrm{NT}$ in a batch reactor for the degradation of Ibuprofen. Ibuprofen (IBU) was examined because it is one of the most common emerging contaminants and is also a good representative for other compounds. To the best of the authors knowledge this is the first report of the use of a non-functionalized $\mathrm{TiO}_{2}-\mathrm{NT}$ array for the electrocatalytic degradation of a pharmaceutical. We have also shown that a modified gold coated $\mathrm{TiO}_{2}-\mathrm{NT}$ array can be used to detect IBU in water without any solution modifications. The development of a combined electrocatalytic degradation and electrochemical detection system would be ideal for inline effluent treatment as these systems are typically robust, chemical free and could be automated for remote operation.

\section{Materials and methods}

\subsection{Formation of the Titanium dioxide nanotube-based arrays}

The anodic formation of the $\mathrm{TiO}_{2}-\mathrm{NT}$ arrays are described in detail in our earlier work (Carlson et al. [5]); however, the following is an overview of the process. A titanium wire coil (ESPI metals, 99\% purity) was used for the degradation array (DA) and titanium foil (ESPI metals, 99\% purity) was used for the sensor array (SA). Nanotube arrays were anodically formed at $30 \mathrm{~V}$ for 1 hour in a fluorinated ethylene glycol solution (96 ethylene glycol, 3.5 DI water and 0.5 ammonium fluoride $\mathrm{wt} \%$ ). 


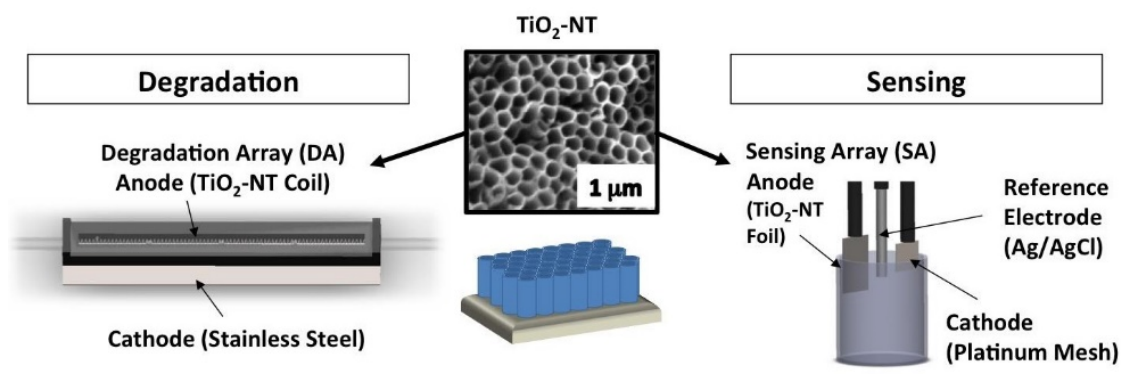

Figure 1: Schematic of the degradation device and sensing setup using a $\mathrm{TiO}_{2}$ NT array as the base material.

Nanotubes formed under these conditions (Figure 1) had average tube diameters and lengths of $70 \mathrm{~nm}$ and $2 \mathrm{um}$, respectively. Crystallization of the amorphous arrays was achieved by annealing at $500^{\circ} \mathrm{C}$ for $2 \mathrm{~h}$; DAs were annealed in $2 \%$ hydrogen (nitrogen balance) to enhance the conductivity of the nanotubes, while the SAs were annealed in oxygen to maximize the resistivity of the nanotubes. Post annealing, the SAs were sputtered with a nm layer of gold to create a hydrophobic surface, as IBU is a non-polar molecule.

\subsection{Fabrication of the degradation array device and sensing array setup}

Figure 1 shows a schematic of the DA device and SA setup. The DA was incorporated into an electrocatalytic cell, with the DA acting as the anode and a stainless steel foil as the cathode. A MakerBot ${ }^{\mathbb{R}}$ Replicator $^{\mathrm{TM}} 2$ was used to build the body of the device from PLA. The single channel reactor $(3 \mathrm{~mm}$ x $230 \mathrm{~mm} \times$ $3 \mathrm{~mm}$ ) held $3 \mathrm{ml}$ of solution and contained a wire coil (with nanotubes on the surface) which had a total surface area of ca. $510 \mathrm{~cm}^{2}$. A multi-channel reactor used in the radical generation testing had a $12 \mathrm{~mL}$ volume with a surface area of ca. $3070 \mathrm{~cm}^{2}$. The SA setup used in this study is composed of the SA anode, a Pt cathode and an $\mathrm{Ag} / \mathrm{AgCl}$ reference electrode.

\subsection{Radical formation and optimization}

Bacterial inactivation in the presence of radical scavenging chemicals was performed to determine the species of radical and relative concentrations generated during the application of an anodic bias to the DA device. Bacteria was used for testing instead of chemicals due to its sensitivity and higher rate of inactivation compared to chemical degradation, and made it easier to determine the presence of radical concentration. In the multi-channel reactor, $6 \mathrm{~V}$ was applied to the system with a flow rate of approximately $50 \mathrm{ml} / \mathrm{min}$ provided a retention time of ca. $60 \mathrm{~s}$. Voltages between 3 and $6 \mathrm{~V}$ were applied to this same reactor under static conditions for $60 \mathrm{~s}$. The chemical scavengers were added to a base solution of $50 \mathrm{ppm} \mathrm{NaCl}$ containing E. Coli 25922 at a concentration of $10^{\wedge} 6$ colony forming units $(\mathrm{CFU}) / \mathrm{ml}$. The following chemicals were added as scavengers: $1 \mathrm{mM}$ isopropanol for hydroxyl radicals, $1 \mathrm{mM}$ sodium pyruvate for 
hydrogen peroxide and $1 \mathrm{mM}$ sodium oxalate to quench surface holes $(\mathrm{VBh}+)$. After treatment, $50 \mu \mathrm{l}$ of solution was collected and diluted 100 -fold before spreading onto a R2A agar medium. The agar plates were incubated at $37^{\circ} \mathrm{C}$ for $24 \mathrm{hr}$ before a visual enumeration of the colony forming units (CFU). Statistical differences for this test, and all subsequent testing, were determined using a student's t-test.

Optimal voltage-current conditions for maximized radical production were determined using DPD (N, N-diethyl-p-phenylenediamine) dye. When exposed to radicals, the colorless DPD dye changes to a magenta color. DPD solution concentrations were $10 \mathrm{mg} / \mathrm{ml}$ to ensure that there was enough dye to prevent the colored species from converting into its colorless form due to excess oxidation. Solutions were held in the single channel DA device at voltages between 1 and $4 \mathrm{~V}$ for $1.5 \mathrm{~min}$. A semi-quantitative value for the radical concentration in solution was obtained by using a UV/Vis spectrophotometer (Shimadzu, UV 3600) to measure the absorbance at $510 \mathrm{~nm}$ after exposure to various voltages.

\subsection{Ibuprofen degradation}

Ibuprofen (Enzo Life Sciences) solutions with concentrations of $30 \mathrm{ppm}$ were made in $\mathrm{DI}$ and $50 \mathrm{ppm} \mathrm{NaCl}$. Although significantly higher than concentrations observed in natural waters, this level was used to ensure accurate quantification using the available characterization techniques. The $\mathrm{pH}$ of the solutions decreased from 6.2 to 4.7 due to the deprotonation of IBU ( $\mathrm{pKa}=4.43$ ).

Based on the results obtained from the radical optimization studies, the timedependent degradation testing was conducted at $3.5 \mathrm{~V}$. Degradation times ranged from $5 \mathrm{~min}$ to $120 \mathrm{~min}$. IBU degradation was monitored using UV/Vis spectrophotometry (Shimadzu, UV 3600) and quantified using chemical oxygen demand (COD, HACH ULR vials). IBU concentrations were calculated from the measured COD values using the following (Boyles [6]):

$$
C O D=C / F W^{*} R M O * 32
$$

where $\mathrm{C}$ is the concentration of oxidizable compound in the sample (mg/L), FW is the formula weight of the oxidizable compound in the sample $(\mathrm{g} / \mathrm{mol})$, and RMO is the ratio of the number of moles of oxygen to the number of moles of oxidizable compound in their reaction to $\mathrm{CO}_{2}$, water, and ammonia.

\subsection{Ibuprofen detection}

Electrochemical detection of IBU in a $50 \mathrm{ppm} \mathrm{NaCl}$ solution was performed using square wave voltammetry (PalmSens, PSTrace4) using the SA setup (shown in Figure 1). A preconditioning step was performed at $0.5 \mathrm{~V}$ for $60 \mathrm{~s}$ before the measurement was taken. The following parameters were used: scan range from $0.5-2 \mathrm{~V}$, step size of $10 \mathrm{mV}, 25 \mathrm{mV}$ amplitude and $1 \mathrm{~Hz}$ frequency. Between tests, the electrodes were rinsed in ethanol and followed by a scan in DI using the same parameters used in testing but with $-0.5 \mathrm{~V}$ during conditioning step. The same initial solutions used for the degradation studies were used for sensing. 
The anodes examined in the sensor array included oxygen annealed nanotubes (BlankNT-SA), oxygen annealed nanotubes coated in gold (AuNT-SA) and gold coated titanium foil (AuTi-SA).

\section{Results and discussion}

\subsection{Radical formation and optimization}

Typically, UV stimulation of $\mathrm{TiO}_{2}$ is necessary in the production of oxidative radical species when in an aqueous environment. During irradiation, UV stimulation induces electron movement from the valence band (VB) to the conduction band $(\mathrm{CB})$, creating a "hole" in the $\mathrm{VB}(\mathrm{VBh}+)$. If electron-hole recombination does not occur, both species can interact with external species trapped at the surface of the NTs. In the pure semiconductor, the position of the lowest empty energy level in the CB and the highest empty energy level in the VB determine the potential of the exchange reactions that can take place (MéndezArriaga et al. [8]). As the VB is situated at more positive potentials, the power of the oxidative reactions is based on location of the $\mathrm{VB}$ edge. $\mathrm{TiO}_{2}$ is considered a good photocatalyst in aqueous solutions as its bandgap energy $(\mathrm{Eg})$ of $3.2 \mathrm{eV}$ can interact directly with the highest available electron donor - water - to produce powerful hydroxyl radicals.

Carlson et al. [5] demonstrated that annealing $\mathrm{TiO}_{2}-\mathrm{NTs}$ in a reducing atmosphere creates such a high concentration of defects that oxidizing species can be generated in the absence of UV irradiation using only an applied bias. This technique was utilized for the inactivation of bacteria in a multi-channel flow reactor where $+6 \mathrm{~V}$ was applied to the $\mathrm{DA}$ to produce the maximum radical generation without causing physical damage to the NTs. Figure 2 shows that, at flow rate of $50 \mathrm{ml} / \mathrm{min}$, complete bactericide was achieved in $60 \mathrm{~s}$ using $6 \mathrm{~V}$ for all solution except for the solution containing $1 \mathrm{mM}$ sodium oxalate. This result indicates that inactivation was the result of direct interactions with the DA coil and not the formation of secondary radical species (e.g., hydroxyl radicals). At $6 \mathrm{~V}$ the current density was between 16 and $21 \mathrm{~mA} / \mathrm{cm}^{2}$; however, water splitting caused by the higher currents was not visible, as any bubbles formed were swept off the surface and/or redissolved into solution.

The static conditions in the DA batch reactors required a different approach to ensure minimal water splitting for maximum radical generation. Bacterial inactivation in the static reactor, shown in Figure 3, was found to be greatest at $5 \mathrm{~V}$ where the current density was ca. $0.004 \mathrm{~mA} / \mathrm{cm}^{2}$. Unlike in the flow cell, the nature of the batch reactor allowed for the formation of additional radical species that could also participate in bactericidal inactivation. No data for $6 \mathrm{~V}$ was reported as enough gas formation occurred to begin to push the solution out of the reactor.

The DPD dye tests were used to semiquantitatively determine the relative concentration of radicals forming using the various parameters. Figure 4 shows the effect of applied voltage and resulting current density on the DPD color change (absorbance) for the single channel batch reactor. The maximum in absorbance, corresponding to maximum radical generation, occurred at current densities 


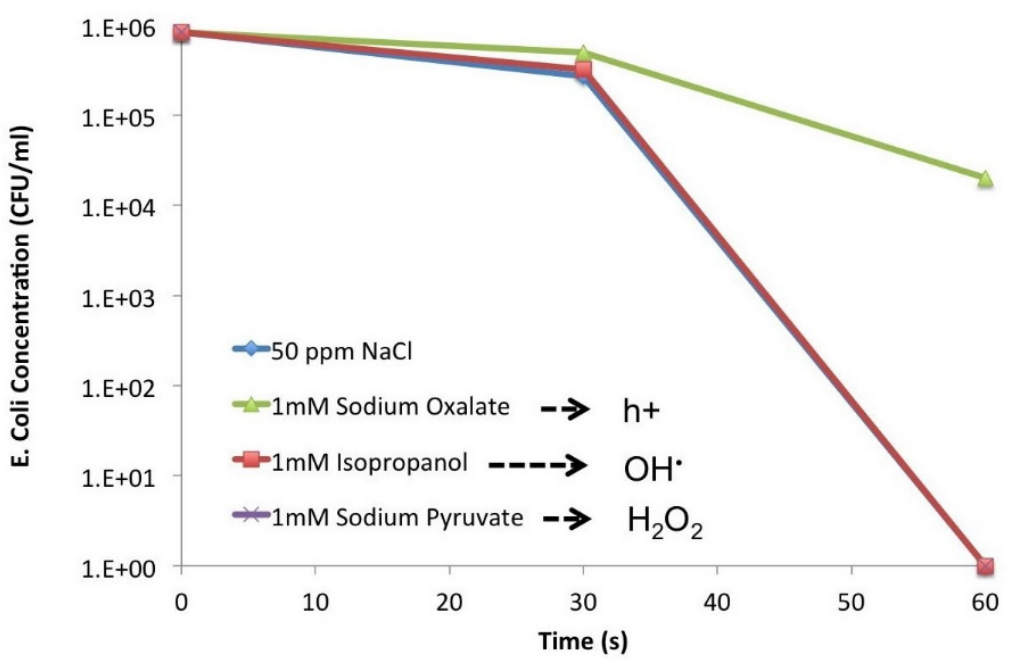

Figure 2: The effects of different radical scavengers on E. Coli inactivation in a $50 \mathrm{ml} / \mathrm{min}$ flow reactor $(60 \mathrm{~s}$ retention time) under an anodic bias of $6 \mathrm{~V}$.

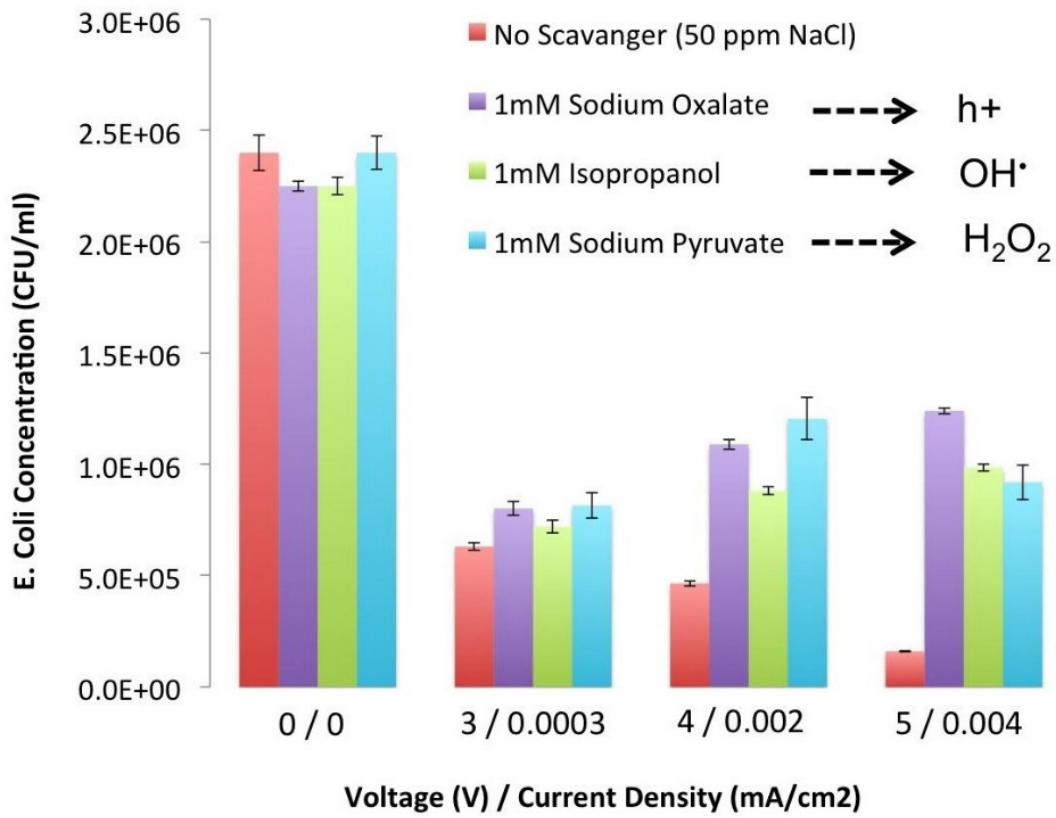

Figure 3: The effects of different radical scavengers on E. Coli inactivation after $60 \mathrm{~s}$ in a batch reactor. 
between ca. $0.002 \mathrm{~mA} / \mathrm{cm}^{2}$. Hydrogen gas evolution at the stainless steel backplate becomes visible at $2.25 \mathrm{~V}$ while oxygen evolution at the DA is not visibly apparent until $3 \mathrm{~V}$. As the voltage is increased, the fine bubbles increase in size and concentration until it begins to force water out of the reactor. Data was not reported for any voltages at which this occurred.

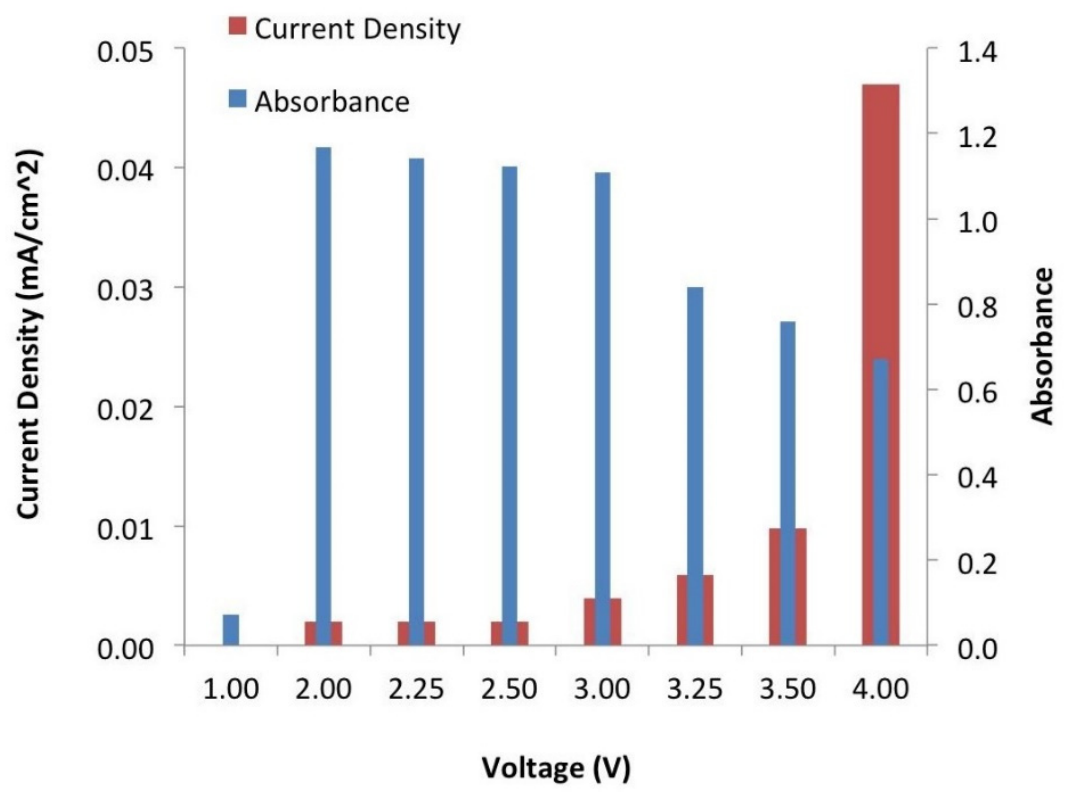

Figure 4: Relationship between radical generation and current density at various applied voltages. High current density means high radical generation.

Several factors are thought to contribute to the lower radical generation when the device is used as a batch reactor instead of a flow reactor, which was its original purpose. The multi-channel reactor used in flow mode at $6 \mathrm{~V}$ produced DPD absorbance values of ca. 0.20 after $1.5 \mathrm{~min}$, corresponding to current densities of ca. $0.30 \mathrm{~mA} / \mathrm{cm}^{2}$ (Carlson et al. [5]). Gas bubbles swept from the surface allowed for the DPD dye to interact directly with the highly oxidizing DA surface. In the batch reactor, the static conditions allow gas bubbles to accumulate on the electrode surface, suppressing radical formation through an overall reduction in the electrode surface area from adsorbed gas.

Testing DPD activity in a beaker under static and stirred conditions confirmed that radical production was impacted primarily by gas adhesion. After applying an anodic 3,4 or $6 \mathrm{~V}$ to the DA for $1.5 \mathrm{~min}$ in a static solution, measured absorbances were $0.10,0.03$, and 0 , respectively. These values did not change significantly until the stir rate was increased enough to retard gas formation at the surface of the electrode, at which the measured absorbances drastically increased to 0.56 , 0.52 , and 0.29 at voltages 3,4 , and 6 , respectively. The lower absorbance values 
for the higher voltages is a result of the greater tendency for cathodic $\mathrm{H}_{2}$ production at the at the stainless steel backplate can also lead to a more reducing environments. Since no agitation can be performed in the channel reactors, the applied bias must be low enough to minimize the effects of water splitting. The use of lower voltages also has an adverse effect on radical production, as lower currents produce to less radical formation. Additionally, the nature of a batch reactor also fosters the formation of weaker radical species, which may not be able to participate in degradation reactions.

\subsection{Ibuprofen degradation}

Figures 5 and 6 show the degradation results for the $30 \mathrm{ppm}$ IBU concentration in solutions of DI and $50 \mathrm{ppm} \mathrm{NaCl}$, respectively. The absorbance spectra of the IBU molecule has a distinct absorbance peak at $220 \mathrm{~nm}$, which becomes obscured and eventually disappears after a 120 min treatment. The peak between 250 and 350 $\mathrm{nm}$ increases with time, as the IBU molecules convert to the various degradation products (Méndez-Arriaga et al. [8], Xiang et al. [9]). A slightly greater concentration of intermediates formed in the $\mathrm{NaCl}$ solution due to the presence of chlorine radicals (Xiang et al. [9]).
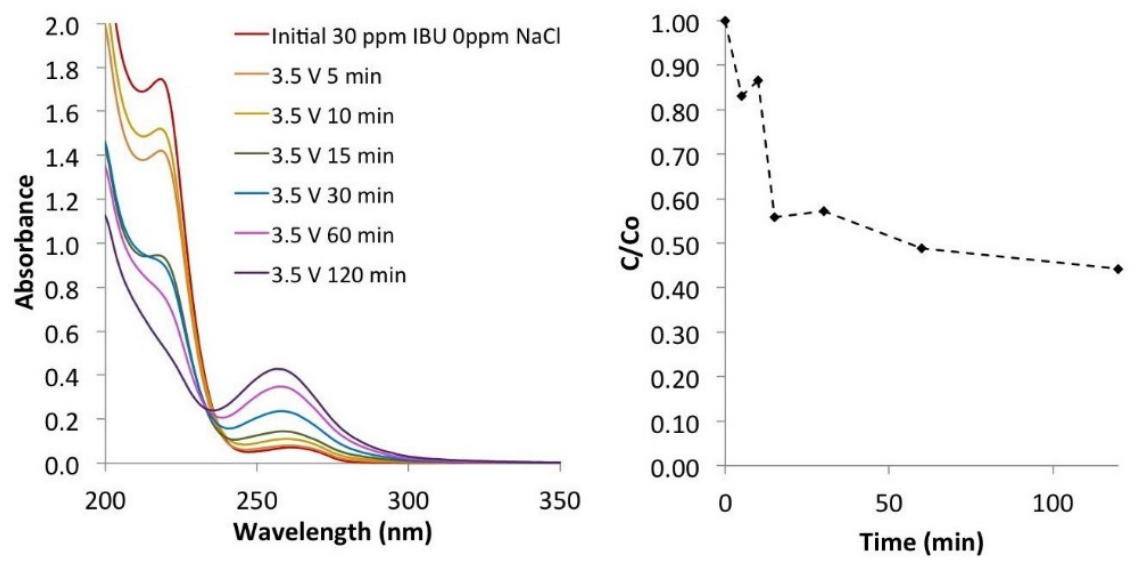

Figure 5: Degradation of $30 \mathrm{ppm}$ IBU with time in DI solution.

The COD values from the DI solution in water decreased over time, degrading to ca. $50 \%$ after $15 \mathrm{~min}$. Hydroxyl radicals are critical to efficient and effective degradation because of their high oxidizing power and unselective attack on organic molecules (Fernando [10]). Attack initializes with hydroxylation, decarboxylation or demethylation of the IBU, followed by the attack and subsequent opening of the benzene ring (Xiang et al. [9]). The plateau in degradation after $15 \mathrm{~min}$ is indicative of the formation of terminal carbon species that cannot be brought to full mineralization i.e. decomposition by oxidation. This result is in line with the bacterial radical scavenging results, which showed the 
formation of weaker radical species in the batch reactor. The oxidizing power of these species may not be great enough to participate in the degradation process.

Degradation in the $\mathrm{NaCl}$ solution could not be quantified due to interference from additional carbon species, which appeared as an increase with the COD values over time. The correlation with the area under the curve is consistent and was repeatable, indicating that the species that were generated were real. As rinsing steps (DI and ethanol) did not show any residuals on the nanotube surface, it is theorized that the additional carbon species resulted from interactions with the $\mathrm{TiO}_{2}$-NT. Nanotubes are formed in ethylene glycol, and upon annealing in a reducing atmosphere, carbon species remain on the surface. Arrays can easily be treated to prevent contamination in a real world system.
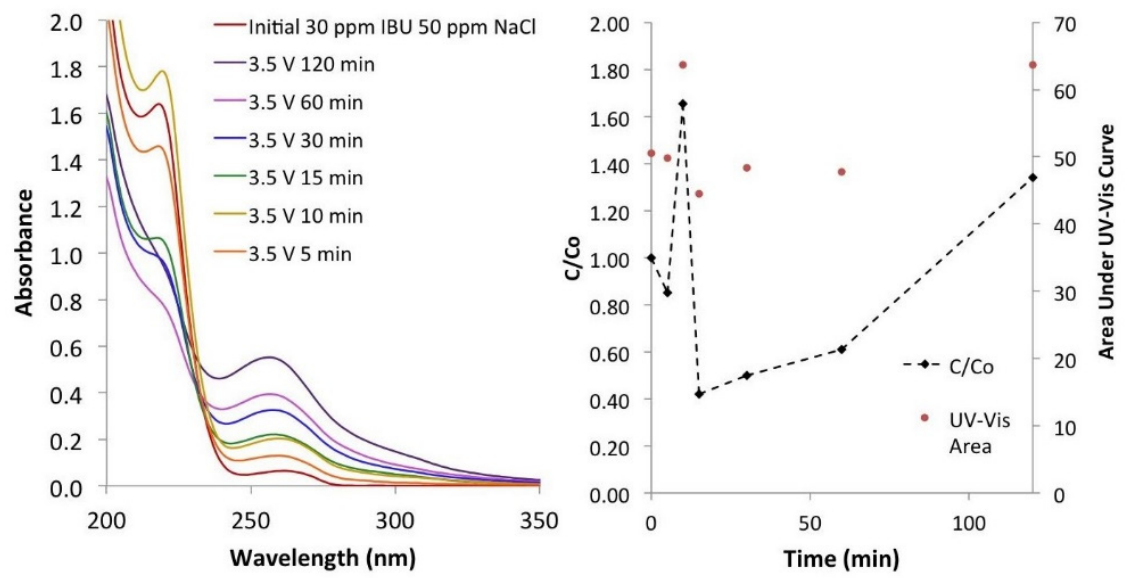

Figure 6: Degradation of $30 \mathrm{ppm}$ IBU with time in $50 \mathrm{ppm} \mathrm{NaCl}$ solution.

\subsection{Sensing}

An ideal point-of-use detection system would have both the selectivity and sensitivity for pharmaceuticals without the need for solution adjustment. Figure 7 confirms the feasibility of this type of system using a gold coated $\mathrm{TiO}_{2}-\mathrm{NT}$ array (AuNT-SA) for the direct detection of $30 \mathrm{ppb}$ IBU in $50 \mathrm{ppm} \mathrm{NaCl}$. A distinct peak can be observed at ca. $0.92 \mathrm{~V}$ corresponding to the oxidation of IBU. The limit of detection for the AuNT was found to be $3 \mathrm{ppb}$. Uncoated, the oxygen annealed NTs (BlankNT-SA) are unable to participate in any type of redox reaction due to the high resistivity of the NTs. The gold coating provides increased conductivity for improved electronic exchange and creates a very hydrophobic surface, enhancing its interaction with the non-polar deprotonated IBU.

Furthermore, the gold shields the negatively charged $\mathrm{TiO}_{2}$ surface from the negatively charged IBU molecules. However, the resistivity of the NTs is critical to accurate detection. The higher conductivity of the AuTi-SA allows more charge exchange with solution species. When the IBU is added to the solution, the overall conductivity increases, making it difficult to clearly observe the IBU oxidation peak. 


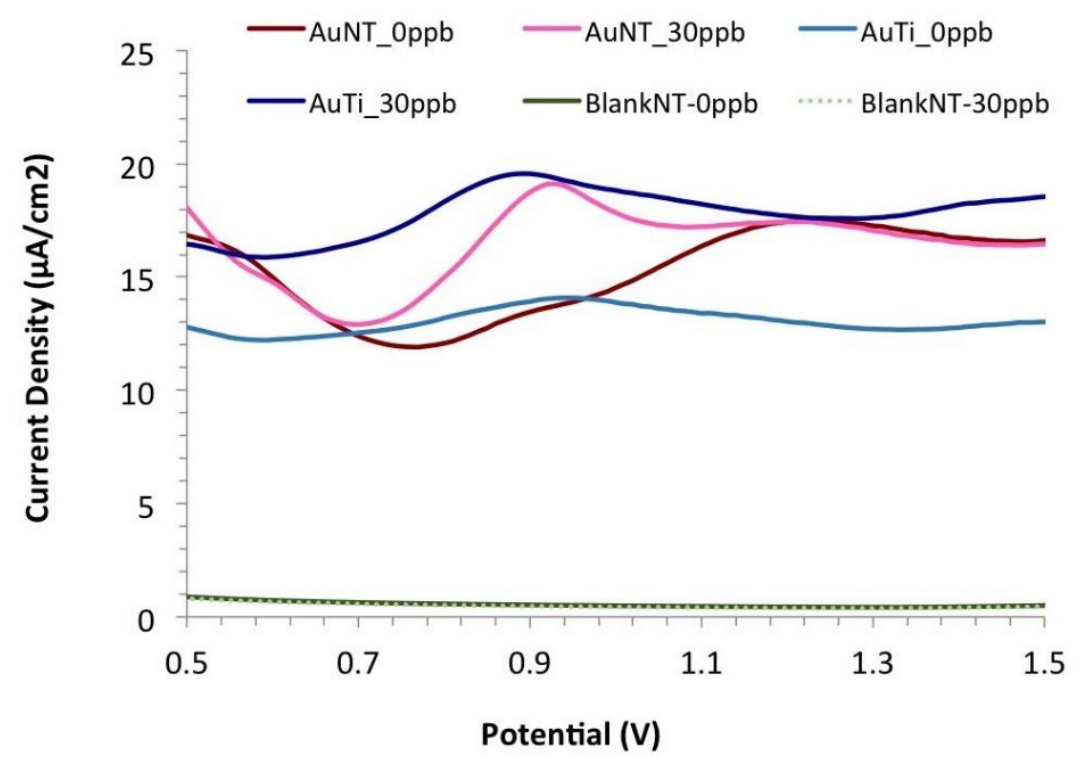

Figure 7: Electrochemical detection of IBU in $50 \mathrm{ppm} \mathrm{NaCl}$ using square wave voltammetry: a) BlankNT-SA, b) AuTi-SA and c) AuNT-SA.

\section{Conclusion}

This study demonstrated both the electrocatalytic degradation and electrochemical sensing of ibuprofen (IBU) in aqueous solutions using modified $\mathrm{TiO}_{2}-\mathrm{NT}$ arrays. Ibuprofen could be degraded by $50 \%$ in 15 min using a $\mathrm{TiO}_{2}-\mathrm{NT}$ array annealed in a reducing atmosphere and detected at levels of $4 \mathrm{ppb}$ using a gold coated $\mathrm{TiO}_{2}$ NT array annealed in oxygen. Batch reactors were less effective than flow reactors at radical generation due to the accumulation of gas on the electrode surface from water splitting.

\section{Acknowledgements}

The authors would like to thank the USTAR and the National Science Foundation (Award 1521433) for their support. We would also like to thank Casey Elliott and Shaikha Abedin for their assistance in testing and sample preparation.

\section{References}

[1] Snyder, S., Westerhoff, P, Yoon, Y D. Sedlak, Pharmaceuticals, personal care products and endocrine disruptors in water: Implication for the water industry. Environmental Engineering Science 20, 449-469 (2003). 
[2] Boleda, M., Galceran, M., Ventura, F., Behavior of pharamaceuticals and drugs of abuse in a drinking water treatment plant (DWTP) using combined conventional and ultrafiltration and reverse osmosis (UF/RO) treatments. Environmental Pollution 159, 1584-1591 (2011).

[3] Clarke, B., Smith, S., Review of 'emerging' organic contaminants in biosolids and assessment of international research priorities for the agricultural use of biosolids. Environment International 37, 226-247 (2011).

[4] Cesaro, A., Naddeo, V., Belgiorno, V., Wastewater treatment by combination of advanced oxidation processes and conventional biological systems. Bioremediation \& Biodegradation 4, 1000208-1000216 (2013).

[5] Carlson, K., Elliott, C., Walker, S., Misra, M., Mohanty, S., An effective, point-of-use water disinfection device using immobilized 'black' TiO2 nanotubes as an electrocatalyst. Journal of The Electrochemical Society, 163 (6) H1-H7 (2016).

[6] Boyles, W. "The Science of Chemical Oxygen Demand. Technical Information Series, Booklet No. 9. Hach Company. 1-24 (1997).

[7] Beranek, R., (Photo)electrochemical methods for the determination of the band edge positions of TiO2-based nanomaterials. Adv. Phys. Chem. (2011).

[8] Méndez-Arriaga, F., Esplugas, S., Giménez, J. Degradation of the emerging contaminant ibuprofen in water by photo-Fenton. Water Research 44. 589595 (2010).

[9] Xiang, Y., Fang, J., Shang, C. Kinetics and pathways of ibuprofen degradation by the $\mathrm{UV} /$ chlorine advanced oxidation process. Water Research 90. 301-308 (2016).

[10] Fernando, B., Ozone reaction kinetics for water and wastewater systems. CRC Press LLC. (2004). 